\title{
Traduire
}

Revue française de la traduction

$232 \mid 2015$

Intraduisible? Vous voulez rire !

\section{Le rire de Samuel Beckett en arabe}

\section{Amani Rabeh}

\section{(2) OpenEdition}

\section{Journals}

Édition électronique

URL : http://journals.openedition.org/traduire/700

DOI : 10.4000/traduire.700

ISSN : 2272-9992

\section{Éditeur}

Société française des traducteurs

\section{Édition imprimée}

Date de publication : 15 juin 2015

Pagination : 86-98

ISSN : 0395-773X

\section{Référence électronique}

Amani Rabeh, «Le rire de Samuel Beckett en arabe », Traduire [En ligne], 232 | 2015, mis en ligne le 15 juin 2017, consulté le 01 mai 2019. URL : http://journals.openedition.org/traduire/700 ; DOI : 10.4000/ traduire.700 


\section{Le rire de Samuel Beckett en arabe}

\section{Amani Rabeh}

"Dis-moi de quoi tu ris, je te dirai qui tu es. "(1)

L'humour traduit l'identité ethno-socio-culturelle de la personne et plus largement d'une communauté d'appartenance qui partage cet imaginaire identitaire collectif. II est difficile dès lors pour un étranger de comprendre la parole humoristique d'un groupe humain s'il ne connait pas les références et les connotations culturelles propres à ce groupe. Le paramètre socioculturel est particulièrement important pour la traduction de l'humour car le traducteur doit faire face à deux défis : rendre tout d'abord compte de manière univoque du cadre culturel de la langue source à travers et par la langue cible ; ensuite, opérer dans la traduction une rupture entre signifiant et signifié, analogue à celle présente dans le texte source. En d'autres termes, il doit adapter le détournement pragmatique et discursif lié au lexique de la séquence humoristique dans le texte source aux structures lexico-syntaxiques de la langue cible.

En attendant Godot et Fin de partie de Samuel Beckett sont les deux pièces les plus célèbres du théâtre de l'absurde où le rire est à la fois posé comme sujet de réflexion et dévoilé à travers de multiples techniques comiques.

II semble que le texte de Beckett, par effet de retournement ironique, tente d'inclure un métadiscours critique sur humour et dérision, ainsi dans l'échange entre Estragon et Pozzo lorsque le premier faisant observer que Vladimir "voit tout en noir", Pozzo lui rétorque : "sauf le firmament. (II rit, content de ce bon mot.) Patience, ça va venir " (EAG, 50)(2).

La démesure des gestes, l'absurdité des situations et des comportements ainsi que les répétitions, les blagues et les jeux de mots, les jeux scatologiques ou sexuels, participent à l'humour sombre et noir beckettien. Le rire accompagne le pessimisme austère et le comique rejoint le tragique chez Beckett qui définit lui-même son théâtre comme révélant : "en face, le pire,

(1) Marcel Pagnol, Notes sur le rire, 1947.

(2) Baron, 1998, p. 83. 
jusqu'à ce qu'il fasse rire „(3). Rire de la condition humaine et traiter du phénomène du rire sont au cœur de son écrit En attendant Godot qui représente réellement un cas troublant et complexe. Si l'humour dans En attendant Godot et Fin de partie est complexe, comment une traduction de ces œuvres en arabe rendrait-elle compte de leur comique "plus désespérant que le tragique " (lonesco)?

Cet article tentera d'analyser la traduction arabe d'En attendant Godot et de Fin de partie élaborée par Paul Chaoul, en particulier la traduction de l'humour verbal vers une langue différente d'un point de vue lexico-syntaxique et dont le destinataire ne partage pas forcément le même langage humoristique ou le même imaginaire ethno-socio-culturel. Comment traduire l'univers burlesque beckettien vers une langue comme l'arabe ? Si le rire est lié à une langue A, peut-on le traduire en une langue B et sinon, quelles solutions s'offrent au traducteur ? Ce sont les questions auxquelles on essayera de répondre ici à travers l'étude des deux textes sources en langue française et de leur traduction en langue arabe. Avant d'entamer l'étude de l'humour verbal beckettien dans la traduction arabe des deux pièces, on abordera la question de la traduction de soi beckettienne et du processus d'écriture-réécriture chez Beckett qui se trouve être à la fois écrivain et auto-traducteur. On proposera ensuite une vision d'ensemble sur la place de l'humour et des effets comiques dans les deux pièces de Samuel Beckett avant d'aborder l'étude de l'humour verbal et des difficultés de sa traduction en arabe.

\section{1 - Quand on traduit pour un auto-traducteur}

Ce n'est qu'en 1953 que Beckett débute sa carrière d'auto-traducteur en revenant à sa langue maternelle après avoir écrit en français de 1946 à 1950, période la plus féconde de sa production, La fin, L'expulsé, Le calmant, Premier amour, Mercier et Camier, Eleuthéria, En attendant Godot et Textes pour rien. La mise en scène d'En attendant Godot par Roger Blin au théâtre de Babylone à Paris et son grand succès auprès du public occidental ont poussé Beckett à traduire, au printemps 1953, son texte en anglais et ainsi éviter les adaptations ratées. De même, Fin de partie, créée en 1957, a d'abord été écrite en français puis traduite en anglais par Beckett lui-même sous le titre Endgame.

La biographie de Beckett témoigne de l'obstination et de la compulsion qu'il avait à traduire ses propres œuvres. L'étude génétique de Solo montre qu'il pouvait reprendre une autotraduction, même après un grand laps de temps. Ce rapport paradoxal entre l'auto-traducteur et son texte à traduire émane de la volonté qu'avait Beckett de recentrer esthétiquement son œuvre d'origine et de l'améliorer. Le procédé de la duplication donne souvent lieu, dans l'œuvre beckettienne, à une révision et plus encore à une autocensure : c'est le cas de First Love où

(3) 'Mirlitonnades', in Collected Poems, p. 66. (Première publication de Poèmes suivi de Mirlitonnades [Paris : Editions de Minuit, 1978]). 
de longs passages ont été coupés. II est fréquent aussi que de cette duplication résulte un enrichissement esthétique. Le texte auto-traduit acquiert chez Beckett une valeur critique.

À la différence d'un traducteur "ordinaire ", Beckett juge son texte à réécrire parfois sans pitié, et en devient le commentateur, voire le censeur selon le cas. Ainsi, tout comme chacun de ses ouvrages répond à des critères différents qui relèvent d'une poétique cohérente, sa pratique d'écriture et de traduction varie selon le regard de l'auteur/sujet à son œuvre selon les diverses contingences : temporelles, linguistiques et, bien évidemment, subjectives. En d'autres termes, Beckett recrée, traduit, réécrit et adapte selon les contraintes du texte et des langues, avec une attention toute particulière au rythme et à la musicalité. De plus, sa pratique d'auto-traducteur diffère selon son rapport à la langue et au regard qu'il porte sur son œuvre au moment où il l'écrit dans l'autre langue(4).

II ne s'agit pas pour nous d'analyser, dans cet article, l'humour verbal dans les traductions anglaises des deux pièces mais dans leur traduction arabe de Paul Chaoul. Ce traducteur, né en 1942 à Beyrouth, au Liban, est poète, critique littéraire et traducteur de poésie française. Outre des recueils de poésie (La boussole du sang, Visage qui sombre ou La mort de Narcisse), des pièces de théâtre (Choukri le suicidaire ou Le visiteur), il est l'auteur d'essais importants tels que La relation du poème moderne avec les arts plastiques et auditifs, (1990). Paul Chaoul a aussi signé une anthologie de poésie française, Le livre de la poésie française contemporaine. Il a également publié deux livres sur la culture maghrébine moderne et l'art dramatique arabe et traduit de nombreux poèmes du français, de l'allemand, et de l'anglais.

\section{2 - L'humour beckettien dans En attendant Godot et Fin de partie}

L'humour a toujours fait partie du caractère et de la plume de Samuel Beckett. " Découvrir le Beckett que ses amis ont bien connu : spirituel, toujours capable de rebondir, qui instinctivement opposait à l'adversité son humour et sa détermination à ne pas lâcher prise(5). "

Le philosophe Henri Bergson étudie dans Le Rire : Essai sur la signification du comique toutes les variétés des effets comiques: le comique des formes, le comique des mouvements, le comique de situation, le comique des mots, le comique de caractère. Pour Bergson, la transposition des idées, dans un ton différent mais en conservant les rapports qu'elles ont entre elles, de leur milieu naturel à un autre nouveau permet de créer un langage comique(6). Bergson note ainsi qu'» on obtiendra un effet comique en transposant l'expression naturelle d'une idée dans un autre ton(7)".
(4) Montini, 2011.
(5) Knowlson, 1999.
(6) Bergson, 1900, p. 54-55.
(7) Bergson, 1900, p. 55. 
Samuel Beckett a su réunir dans son théâtre tous les éléments du rire théorisés par Bergson. La charge comique permet à l'œuvre de Beckett de redémarrer chaque fois que celle-ci est, selon ses propres dires, sur le point d'expirer. L'analyse des répliques et des didascalies dans En attendant Godot et Fin de partie témoigne d'une richesse dans l'usage des différentes formes du comique qui se rejoignent et se complètent. De la même manière, Arsène, dans le roman Watt de Beckett distingue trois formes de rires:

L'amer, le jaune et le - ha! - sans joie. Le rire amer rit de ce qui n'est pas bon, c'est le rire éthique. Le rire jaune rit de ce qui n'est pas vrai, c'est le rire judiciaire. Pas bon! Pas vrai ! Enfin ! Mais le rire sans joie est le rire noétique, par le groin - ha! - comme ça, c'est le rire des rires, le "risus purus", le rire qui rit du rire, hommage ébahi à la plaisanterie suprême, bref le rire qui rit - silence s'il vous plait - de ce qui est malheureux(8).

Le rire beckettien qui caractérise les deux pièces En attendant Godot et Fin de partie est essentiellement un risus purus, c'est-à-dire un rire qui rit du malheur, du contexte misérable ou grave comme quand Vladimir ajoute dans En attendant Godot, concernant le projet de se suicider en se jetant de la tour Eiffel : « Maintenant il est trop tard. On ne nous laisserait même pas monter".

\section{3 - Traduction arabe de l'humour verbal beckettien}

Très présent dans les deux pièces de Beckett, le comique de mots est souvent lié aux autres effets comiques de situation et de circonstance. Bergson note qu' « il y a peut-être quelque chose d'artificiel à faire une catégorie spéciale pour le comique de mots, car la plupart des effets comiques que nous avons étudiés jusqu'ici se produisaient déjà par l'intermédiaire du langage(9)".

Le vocabulaire total des deux pièces compte 2000 mots(10), ce qui témoigne d'une richesse lexicale considérable. Le champ lexical du comique sous sa forme adjectivale, nominale et verbale (comique, drôle, rire) présent abondamment dans les deux pièces a été majoritairement traduit dans le texte de Paul Chaoul par la forme yazhak (littéralement : il rit) et ses dérivés : muzhhik " drôle ", zahịik " rire " et yuẓhik « il fait rire ". Le nom " comédie " a reçu deux traductions arabes : mash ara (litt : sujet de risée, bouffonnerie) et mahzala (litt : farce).

Bergson distingue le comique verbal traduisible du comique verbal intraduisible. II écrit ainsi :

Il faut distinguer entre le comique que le langage exprime et celui que le langage crée. Le premier pourrait, à la rigueur, se traduire d'une langue dans une autre, quitte à perdre la plus

(8) Beckett, 1987, p. 48-49.

(9) Bergson, 1900, p. 47.

(10) Phalèse, 1998, p. 40. 
grande partie de son relief en passant dans une société nouvelle, autre par ses mœurs, par sa littérature, et surtout par ses associations d'idées. Mais le second est généralement intraduisible. II doit ce qu'il est à la structure de la phrase ou au choix des mots. II ne constate pas, à l'aide du langage, certaines distractions particulières des hommes ou des événements. II souligne les distractions du langage lui-même. C'est le langage lui-même, ici, qui devient comique (11).

Samuel Beckett a eu recours aux deux types de comique des mots. À nous de voir quels compromis a trouvé Paul Chaoul pour contourner les difficultés liées au comique verbal intraduisible ou difficilement traduisible.

\section{1 - Jeux des assonances et répétitions phoniques}

Dans sa création de jeux de mots et d'humour verbal, Samuel Beckett a une prédilection pour les répétitions phoniques et pour les jeux d'assonances. La répétition phonique caractérise d'emblée les diminutifs affectueux " Didi " et "Gogo " qui, en usage, donnent naissance parfois à des assonances risibles et enfantines :

ESTRAGON (avec effort) - Gogo léger, branche pas casser, Gogo mort, Didi lourd, branche casser, Didi seul [...] (EAG, 21).

Paul Chaoul traduit littéralement ces séquences en remplaçant le son [g] qui n'existe pas en arabe littéral par le son [j] ce qui donne :

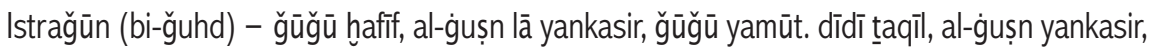
dīdī yabqā waḥdah. [...] $(2009,53)$.

Une cacophonie plaisante née d'un jeu d'assonances s'installe lorsqu'Estragon invite Vladimir à parler en lui disant :

ESTRAGON (avec douceur) - Tu voulais me parler? (Vladimir ne répond pas. Estragon fait un pas en avant.) Tu avais quelque chose à me dire? (silence; autre pas en avant) Dis, Didi...

VLADIMIR (sans se retourner) - Je n'ai rien à te dire. (EAG, 20)

Le jeu phonétique construit sur le triplement du son [di] et sur l'homophonie entre l'impératif " dis " et le diminutif affectueux " Didi " apparente la séquence à un énoncé impératif où le verbe " dis " serait triplement employé. La traduction arabe ne peut rendre compte de ce jeu phonétique car le verbe " dis " se traduit en arabe par " qul " : Qul yā dīdī $(2009,51)$, ce dont atteste le texte de Paul Chaoul.

(11) Bergson, 1900, p. 47-48. 
Lorsque la répétition phonétique concerne un mot ou une séquence phonétique à forte connotation culturelle, Paul Chaoul procède généralement à une modulation ou à une explicitation de l'énoncé :

ESTRAGON (avec volupté) - Calme...Calme... (rêveusement) Les Anglais disent câââm. Ce sont des gens câââms. (un temps) Tu connais l'histoire de l'Anglais au bordel ? (EAG, 19)

Istraǧūn (bi-našwa). - hudū'...hudū'... (ḥāliman) al-inklīz yugianniǧūn fĩ qawl hudū'... hudū' (șamt). hal ta'rif ḥikāyat al-inkilīzī alladī dِahaba ilā I-māhūr ? $(2009,50)$

L'énoncé " les Anglais disent câââm " dénote une charge socioculturelle. Très probablement afin de faciliter sa compréhension par le lecteur arabe qui ne connaît pas forcément la culture et le parler anglais, Paul Chaoul procède à un rajout de sens en traduisant littéralement : les Anglais chouchoutent le mot " calme " quand ils le prononcent.

Dans sa traduction des jeux phoniques, Paul Chaoul fait parfois le choix de passer sous silence certains énoncés. C'est le cas de" ce sont des gens câââms " qui, appauvri sémantiquement et lexicalement, se trouve réduit dans la traduction arabe au terme hudū' "calme ". À noter ici que dans sa traduction anglaise de la pièce, Samuel Beckett lui-même n'a pas traduit cette séquence :

ESTRAGON: (voluptuously) Calm ... calm ... The English say cawm. (pause) You know the story of the Englishman in the brothel?

\section{2 - Paronomases et calembours}

Samuel Beckett use abondamment dans ses deux pièces de paronomases et de calembours. Ces procédés langagiers contribuent largement à l'aspect comique. Les paronomases sont un rapprochement effectué entre des termes voisins par leur assonance mais différents par leur signifié. Ainsi, certaines paronomases sont construites sur un jeu langagier anglophone :

HAMM (fièrement) - Sans moi (geste vers soi), pas de père. Sans Hamm (geste circulaire), pas de home. (FDP, 54)

La traduction arabe n'a pu conserver ce rapprochement phonique entre "Hamm " et " home " dans la mesure où " home " a été traduit par bayt, alors que la dénomination a été traduite phonétiquement. Toutefois, Paul Chaoul a essayé de garder un certain parallélisme dans la construction syntaxique des deux propositions.

Hamm -(bi-'tizāz) (išāra ilā nafsih) kunta bilā abb bi-dūn hamm (ḥaraka dāiriyya). kunta bilā bayt ! (1992, 53)

Littéralement :

Hamm - (fièrement) (geste vers soi) Sans Hamm, tu étais sans père (geste circulaire). Tu étais sans maison! 
Samuel Beckett donne une tout autre traduction de la réplique dans Endgame. L'auto-traduction est, comme on l'a vu précédemment, en elle-même un processus de réécriture pour Beckett :

HAMM (proudly): But for me, (gesture towards himself) no father. But for Hamm, (gesture towards surroundings) no home.

Cet effet humoristique lié aux paronomases n'a généralement pas été rendu dans la version arabe. Cela est logique dès lors que l'on sait que les paronomases sont des jeux qui naissent des propriétés phoniques propres aux termes d'une langue donnée. Ces propriétés disparaissent quand on passe d'une langue à une autre.

CLOV (regardant toujours) - Le fanal est dans le canal. (FDP, 45)

Klūf(12) : ḍaw' al-manāra. [Littéralement : lumière du phare] $(1992,46)$

VLADIMIR (à Estragon) - Fais voir. (Estragon lui montre sa jambe ; à Pozzo, avec colère). II saigne!

P0ZZO. - C'est bon signe. (EAG, 41)

Fladimīr - (ilā istraǧūn) arinī! (istraǧūn yurīhi sāqahu. ilā pūzzū, bi-ġaḍab) innahu yanzif. Pūzzū - hādihi 'alāma jayyida. (2009, 76)

En traduisant " signe " par 'alāma et " saigne " par yanzif, la version arabe n'a pu conserver le rapprochement phonique présent en français. L'humour, qui n'est pas porté dans la traduction arabe par les paronomases, naît souvent du comique de l'ensemble de la situation qui devient caricaturale et risible.

Lorsqu'on analyse le comique des mots chez Samuel Beckett, on ne peut que constater le foisonnement de calembours dans son texte. Ce procédé permet de créer une sorte de jeu de devinettes entre Samuel Beckett et le lecteur et participe au caractère comique des pièces. Tout comme le procédé des paronomases, les calembours font partie des jeux langagiers intraduisibles, ce qui rend la tâche de la traduction de cet humour verbal en arabe très complexe, voire impossible.

Pozzo. - II s'apaise. (regard circulaire) D'ailleurs, tout s'apaise, je le sens. Une grande paix descend. Écoutez. (il lève la main) Pan dort. (EAG, 46)

Būzzū - hadda' (yanẓur ḥawālayh) 'alā kul hāal yabdū anna kul šay' hada'. Sukūn 'aẓīm yasūd. isma'ā. (yarfa' yadah) Bān yanām. $(2009,81)$

Littéralement :

Pozzo - II s'est calmé (il regarde autour de lui) D'ailleurs, on dirait que tout est calme. Un immense silence règne. Écoute. (il lève la main) Pan dort.

(12) Traduction inexistante en arabe de la didascalie. 
Les calembours sont connus des lecteurs de la langue source et font partie de leur imaginaire identitaire collectif. Dès lors que l'on sort de cet imaginaire ou si l'on ne possède pas d'équivalent dans la langue cible, le calembour ne peut être reproduit ni compris par les lecteurs de la langue cible. Dans sa traduction arabe de l'expression poétique de Pozzo faisant allusion à la boite de Pandore, Paul Chaoul n'avait d'autre choix que de traduire littéralement "Pan dort " par Bān yanām (sujet + verbe).

La traduction de ce genre d'énoncé devient encore plus complexe lorsque le calembour joue, dans le texte source, sur l'usage de deux consonnes dont une qui n'existe pas dans la langue cible. En arabe littéral, il n'existe pas de lettre équivalente à la lettre " $P$ ", qui est rendue par un son [b]. Le dialogue comique suivant est construit sur le jeu consonantique entre la lettre " $B$ " et la lettre " $P$ ". Dans la traduction arabe de Paul Chaoul, les deux consommes sont rendues de manière équivalente par la lettre " ب" qui reprend le son [b] : le jeu consonantique est donc inexistant dans la version arabe, tout comme la charge humoristique portée par l'écriture en lettres capitales de la réplique de Pozzo car l'arabe est une langue monocamérale, qui ne connaît pas de majuscules.

ESTRAGON (faisant semblant de chercher) - Bozzo... Bozzo...

VLADIMIR (de même) - Pozzo...

POZZO. - PPPOZZO !.

ESTRAGON. - Ah ! Pozzo ... voyons... Pozzo ...

VLADIMIR. - C'est Pozzo ou Bozzo?

\section{3 - Néologismes, mots-valises et synonymie}

Dans l'élaboration de l'effet humoristique global, Samuel Beckett n'hésite pas à inventer au besoin des néologismes comme "Knouk " que Paul Chaoul emprunte et arabise dans sa traduction $k n u ̄ k(2009,77)$. Le traducteur a sans doute préféré mettre en relief la nature du mot dans le texte source pour rendre au mieux le style d'écriture de Samuel Beckett.

À côté des véritables néologismes, peu fréquents dans les deux pièces de Samuel Beckett mais utilisés pour leur effet comique, on dénombre quelques mots-valises:

ESTRAGON. - Mais non, je n'ai jamais été dans le Vaucluse ! J'ai coulé toute ma chaudepisse d'existence ici, je te dis ! Ici ! Dans la Merdecluse ! (EAG, 80)

Istraǧūn - lā lam akun ițtāqan fī l-fūklūz. qultu laka innī amḍaytu ḥayātī I-ḥaqīra hunā !hunā ! (2009, 118)

Dans cet exemple, Paul Chaoul traduit uniquement le mot composé " chaude-pisse " et fait abstraction du mot-valise "Merdecluse ". Dans sa traduction de " chaude-pisse " en arabe, il 
procède à une explicitation du signifié : il reformule l'idée dans un terme existant dans la langue d'arrivée. Pour dire que la vie d'Estragon n'était pas bien rose, Paul Chaoul emploie l'expression hayātī al-ḥaqīra (ma vie méprisable). La création verbale "Merdecluse " a un aspect scatologique. C'est probablement la raison pour laquelle Paul Chaoul a omis de la traduire. Cependant, une explicitation de ce mot-valise pouvait aussi fonctionner en arabe, avec toutefois un allègement sémantique : la Merdecluse $\rightarrow$ füklūz al-karīha (le désagréable Vaucluse).

La synonymie est un principe qui participe au comique verbal beckettien. L'emploi des synonymes de manière successive traduit la confusion discursive dans laquelle baignent les personnages.

Pozzo. - Qu'est-ce que j'ai fait de ma pipe ? (EAG 44)

Pozzo. - Mais qu'ai-je donc fait de ma bruyère !

Estragon. - II est marrant il a perdu sa bouffarde !

Pozzo. - J'ai perdu mon Abdullah! (EAG 45)

Les mots " pipe ", " bruyère ", " bouffarde ", " Abdullah " représentent les différentes appellations d'un même objet : 'pipe' est l'appellation neutre, 'bruyère' est une métonymie qui renvoie à la matière dont est faite la pipe (en anglais, Pozzo parle de briar) et 'bouffarde' est le terme argotique (en anglais, Estragon parle de dudeen qui est le terme irlandais pour pipe) ; alors que 'Abdullah' est le nom d'une marque (qui en anglais devient une marque anglaise Kapp and Peterson).

La traduction arabe ne rend pas compte de cette variation de registres (soutenu/argot) et des connotations culturelles (Abdullah devenu en anglais Kapp and Peterson). En effet, Paul Chaoul a choisi de traduire toutes ces variantes du même mot par le même terme neutre ġalyūn (pipe).

\section{4 - Expressions familières et maximes détournées}

La marginalité des personnages de Beckett, une marginalité du neutre, prend des formes multiples notamment dans la variété du vocabulaire qu'ils emploient. Discours respectueux et soutenu ainsi que blasphèmes ou termes familiers et populaires s'entrelacent souvent dans le texte beckettien, créant une confusion rhétorique et un climat ironique. Les termes familiers ou populaires sont directement liés à l'imaginaire socio-culturel d'une communauté, il serait intéressant de voir de plus près la traduction arabe de cette variété du vocabulaire à travers des exemples :

(1) "Le salaud! La vache ! (EAG 41) $\rightarrow$ al-khinzïr! al-hayawān! $(2009,76)$ [litt : Porc ! Animal !]

(2) "Salaud !... Fumier! Crapule ! " (EAG 95) $\rightarrow$ qadir!... nadl! waǵd! $(2009,136)$ [traduction littérale] 
(3) "Cochon !... porc!" (EAG 95) $\rightarrow$ hinzirr! $(2009,136)$ [en arabe, c'est la seule traduction possible pour les deux termes]

(4) "Qui a pété ? " (EAG 106) $\rightarrow$ man ḍarața? $(2009,149)$ [traduction littérale]

(5) "Dis-lui de la boucler! Casse-lui la gueule " (EAG 107) $\rightarrow$ askithu! hatțim fakkahu! $(2009,151)$ [litt : Fais-le taire ! Casse-lui la mâchoire !]

(6) "Mômes " (FDP 102) $\rightarrow$ ațfāl $(1992,87)$ [traduction littérale]

(7) "Putain » (FDP 94) $\rightarrow$ al-āhira (1992, 82) [traduction littérale]

(8) "Je t'en fous " (FDP 46) $\rightarrow$ al-la'na $(1992,47)$ [litt : malheur]

Tel que le montrent ces exemples, Paul Chaoul a procédé généralement à des traductions littérales assez soutenues des expressions familières lorsque celles-ci avaient une traduction en arabe. Quand il n'était pas en mesure de faire des traductions littérales, le traducteur a proposé des équivalences dans la langue d'arrivée comme l'atteste l'exemple (5). Paul Chaoul réalise parfois des modifications sémantiques telles que le montrent les exemples (1) et (8). La traduction du premier terme est un choix fait par Paul Chaoul, pour lequel on n'a pas vraiment d'explication car le même terme reçoit une autre traduction dans l'exemple (2) à savoir " qadir ", l'équivalent le plus proche en arabe.

La langue arabe est marquée par une importante diglossie entre l'arabe littéraire et l'arabe dialectal ou parlé. Les expressions familières sont surtout recensées dans la langue parlée mais Paul Chaoul ne pouvait pas puiser dans les dialectes arabes car le registre familier diffère selon les pays. Paul Chaoul tenait très probablement à ce que sa traduction soit lue par la plupart des lecteurs arabes, ce qui explique son choix de faire usage d'un style soutenu compris dans tous les pays arabes.

L'humour verbal repose également chez Beckett sur l'utilisation détournée des maximes et des sentences. Beckett aime inverser les termes dans les locutions figées, ce qui entraîne un détournement du sens initial des formules.

(1) "Si vieillesse savait ! ॥ (FDP 22) $\rightarrow$ law 'arifat al-šayhūhna! (1992, 28) [traduction littérale]

(2) "Du tic au tac " (EAG 48) $\rightarrow$ cette formule, allusion à " tac au tac " et comprise dans les didascalies, n'a reçu aucune traduction dans la version de Paul Chaoul.

(3) "Quand on cherche on entend " (EAG 82) $\rightarrow$ 'indamā yabḥat al-insān yasma' (2009, 121) [litt : Quand l'Homme cherche, il entend].

Le premier exemple tiré de Fin de partie est le détournement du proverbe « si jeunesse savait, si vieillesse pouvait ". Paul Chaoul propose une traduction littérale, qui fait en même temps référence à un roman célèbre intitulé law 'arifa al-šabāb (Si la jeunesse savait) de Tawfīq al-Hakīm, écrivain égyptien et grande figure des lettres arabes, à la fois pionnier de l'écriture théâtrale et nouvelliste. 
Le troisième exemple tiré d'En attendant Godot a été également rendu par une explicitation par ajout de sens lié à l'introduction du terme insān (homme) en tant qu'espèce, opposé en arabe au terme hayawān (animal). Paul Chaoul aurait pu, afin de garder la nature de l'expression, c'est-à-dire la parole proverbiale, faire appel au proverbe arabe man yabhat yajid, qui n'est autre que la traduction arabe du proverbe de Sophocle (Qui cherche trouve). En traduisant l'expression beckettienne par man yabhat yasma' (Qui cherche entend), on conserve le caractère proverbial de l'expression initiale par détournement d'un proverbe déjà connu du lecteur arabe.

\section{Conclusion}

L'humour beckettien est un humour complexe qui repose sur une assise comique très variée. Les jeux de mots, très utilisés dans les deux pièces En attendant Godot et Fin de partie, contribuent largement à l'élaboration des effets comiques. L'humour verbal beckettien use, comme on vient de le voir, des jeux phoniques (répétitions, calembours, paronomases) mais aussi des jeux sur le sens des termes (niveaux de langue et néologismes). L'objectif de cet article était de vérifier si ce travail sur le son et sur le sens était transposable en arabe et le cas échéant, quels étaient les compromis trouvés par Paul Chaoul pour contourner ces difficultés de traduction.

Il est évident, après l'étude du texte source et du texte cible, que la traduction arabe de l'humour verbal dans ces deux pièces repose essentiellement sur un travail sur le sens. Les effets produits par les rapprochements phoniques n'ont généralement pas pu être rendus dans la version arabe du texte car les caractéristiques phoniques des termes ont changé lors du passage d'une langue à l'autre. Lorsque le comique langagier reposait sur une création verbale, Paul Chaoul a adopté l'une des deux attitudes suivantes : emprunter et arabiser le terme ou le passer sous silence. La traduction des tournures détournées ainsi que celle des termes familiers et populaires en arabe ne s'est pas faite sans difficulté. Maximes, sentences et mots familiers font référence à un imaginaire socioculturel. Le lecteur arabe qui ne partage pas forcément ce même imaginaire ne peut apprécier ce détournement de sens des maximes et des proverbes et partager cet humour intra-langagier dont Samuel Beckett a su tirer le meilleur parti.

L'humour, dans les traductions arabes de Paul Chaoul, repose principalement sur le comique de situation, du geste et du mouvement ainsi que sur le comique de caractère. Le comique des mots est traduisible lorsqu'il est exprimé par le langage mais ne peut être traduit lorsqu'il naît du langage. Paul Chaoul a ainsi pu traduire la dénotation des jeux de mots mais non leurs connotations qui font directement appel aux références culturelles ou aux registres de langue. Face à la complexité de la traduction de ce genre d'humour, les stratégies de Paul Chaoul vont de la norme rédactionnelle la plus conformisante à la plus différenciatrice. 


\section{Bibliographie :}

BARON Christine, 1998, Samuel Beckett, "En attendant Godot » et "Fin de partie », Paris, Ellipses. BECKETT Samuel, 1952, En attendant Godot, Paris, les Éditions de Minuit.

BECKETT Samuel, 1957, Fin de partie, Paris, les Éditions de Minuit.

BECKETT Samuel, 1992, Fin de partie, trad. arabe par Paul Chaoul, Kuweït, Dār al-kutub bi-wizārat al-i'lām.

BECKETT Samuel, 2009, En attendant Godot, trad. arabe par Paul Chaoul, Beyrouth, Manšūrāt al-Jamal.

BECKETT Samuel, 1987, Watt, Paris, Minuit.

BERGSON Henri, 1900, Le rire. Essai sur la signification du comique, Québec, Université du Québec à Chicoutimi, http://dx.doi.org/doi:10.1522/cla.beh.rir, consulté le 28 mars 2015.

COHN Ruby, 1962, Samuel Beckett: the comic gamut, New Brunswick, Rutgers University Press. COLLINGE Linda, 2000, Beckett traduit Beckett [Texte imprimé] : de Malone meurt à Malone dies : I'imaginaire en traduction, Genève, Droz.

KNOWLSON James, 1999, Beckett, Paris, Solin Actes Sud.

LAURIAN Anne-Marie et SZENDE Thomas (dir.), 2001, Les mots du rire : comment les traduire? Essais de lexicologie contrastive, Berne, P. Lang.

MONTINI Chiara, 2011, Le rôle du bilinguisme de Beckett dans la genèse de Mercier and Camier, Paris, Institut des textes et manuscrits modernes (Item), http://www.item.ens.fr/index.php?id=577789, consulté le 7 avril 2015.

PHALESE Hubert de, 1998, Beckett à la lettre : "En attendant Godot ", "Fin de partie ", Paris, Nizet.

SARDIN Pascale, 2002, Samuel Beckett auto-traducteur ou l'art de "l'empêchement " : lecture bilingue et génétique des textes courts auto-traduits : 1946-1980, Arras, Artois presses université.

SCHWERTER Stéphanie et K. DICK Jennifer (dir.), 2013, Traduire : Transmettre ou trahir ? : Réflexions sur la traduction en sciences humaines, Paris, Éditions de la Maison des sciences de l'homme.

WOOD Mary Patricia et al., 1999, Humour, culture, traduction(s), Lille, Université Charles-deGaulle. 
Amani RABEH, Docteur en Sciences du langage, s'intéresse à la linguistique contrastive et à la traduction arabe-français. À partir de corpus bilingues variés (littéraire, médiatique), ses recherches visent à analyser aussi bien le texte traduit que le processus de traduction. Elle considère le texte en tant que produit de la traduction et les éléments contextuels qui entrent dans l'activité traduisante pour tenter de repérer les stratégies traductives ainsi que les principes et les phénomènes récurrents dans l'activité de traduction. Ses travaux visent par ailleurs également à étudier des particularités linguistiques et stylistiques liées aux langues française et arabe dans un cadre contrastif. 